\title{
The accuracy of pathological data for the prediction of insignificant prostate cancer
}

Betina Katz, Miguel Srougi, Luiz H. Camara-Lopes, Alberto A. Antunes, Luciano Nesrallah, Adriano Nesrallah, Marcos Dall'Oglio, Katia R. M. Leite

Laboratory of Medical Research - LIM55, Department of Urology, University of São Paulo Medical School (BK, MS, AAA, LN, AN, MD, KRML) and Laboratory of Surgical and Molecular Pathology - Sirio Libanes Hospital (BK, CL, KRML), São Paulo, Brazil

ABSTRACT

Introduction: The widespread screening programs prompted a decrease in prostate cancer stage at diagnosis, and active surveillance is an option for patients who may harbor clinically insignificant prostate cancer (IPC). Pathologists include the possibility of an IPC in their reports based on the Gleason score and tumor volume. This study determined the accuracy of pathological data in the identification of IPC in radical prostatectomy (RP) specimens.

Materials and Methods: Of 592 radical prostatectomy specimens examined in our laboratory from 2001 to 2010, 20 patients harbored IPC and exhibited biopsy findings suggestive of IPC. These biopsy features served as the criteria to define patients with potentially insignificant tumor in this population. The results of the prostate biopsies and surgical specimens of the 592 patients were compared.

Results: The twenty patients who had IPC in both biopsy and RP were considered real positive cases. All patients were divided into groups based on their diagnoses following RP: true positives $(n=20)$, false positives $(n=149)$, true negatives $(n=421)$, false negatives $(n=2)$. The accuracy of the pathological data alone for the prediction of IPC was $91.4 \%$, the sensitivity was $91 \%$ and the specificity was $74 \%$.

Conclusion: The identification of IPC using pathological data exclusively is accurate, and pathologists should suggest this in their reports to aid surgeons, urologists and radiotherapists to decide the best treatment for their patients.
ARTICLE INFO

\section{Key words:}

Prostate cancer; Prostate;

Gleason score; Diagnosis

Int Braz J Urol. 2012; 38: 760-8

Submitted for publication:

February 14, 2012

Accepted after revision:

August 14, 2012

\section{INTRODUCTION}

The detection of patients with nonpalpable prostate cancer has increased since the advent of prostate-specific antigen (PSA) screening. Modified prostatic biopsy schemes have also contributed to higher detection rates (1). Many of these earlier, smaller cancers are low volume $\left(<0.5 \mathrm{~cm}^{3}\right)$, low grade and clinically insignificant tumors. Approximately one third of patients with stage T1c cancer have potentially clinically insignificant tumors, and approximately 5\% of radical prostatectomy (RP) patients have small cancers that are difficult to identify histologically (2).

Epstein et al. initially created a set of four criteria to predict insignificant prostate cancer prior to definitive therapy: PSA density $0.1-0.15$, low or intermediate cancer grade, core involvement of less than $3 \mathrm{~mm}$, and involvement of only one needle biopsy core. These criteria identified 
$79 \%$ of tumors with a volume $\leq 0.5 \mathrm{~cm}^{3}$ that were organ confined and did not qualify as high-grade lesions at the time of RP (3).

However, the Epstein criteria, which were later updated (4), are insufficient, and 20\% of patients who fulfill these criteria may have unfavorable pathological cancer characteristics at RP (5). The validity of these criteria has been questioned. Jeldres et al. demonstrated that the Epstein criteria may not be applicable to European men because prostate cancer was underestimated in $24 \%$ of these patients (1).

The aim of this study was to assess the prediction of insignificant prostate cancer based on the biopsy findings of patients who underwent RP and exhibited insignificant cancer. The biopsy features from these subjects were evaluated, and patients whose biopsies had similar parameters were selected. The biopsies were correlated with the respective RP specimens to identify the lesion characteristics and the clinical significance of the tumor.

\section{MATERIALS AND METHODS}

A total of 592 patients underwent transrectal ultrasound-guided (TRUS) prostate biopsy followed by radical prostatectomy for prostate cancer from January 2001 to December 2010. A single surgeon (MS) treated all patients and a single uropathologist (KRML) examined all biopsies and surgical specimens. The surgical specimens were fixed in 10\% buffered formalin, the entire surgical margin was stained with India ink, the left and right lobes were separated, $3 \mathrm{~mm}$ transverse serial sections were taken from each lobe, and the entire gland was submitted for histologic examination. Sections of the bladder neck, prostatic apex, seminal vesicles, and pelvic lymph nodes were also submitted to exam. The Gleason score (GS) was used for histologic grading (6). The tumor volume was evaluated as described by Humphrey et al. (7). Briefly, a grid was placed below the slides, on which the area involved by the tumor had been previously sketched out. The percentage of tumor on a slide was determined by dividing the number of squares involved by tumor by the number of squares occupied by the whole section on the slide. Tumor volume was defined as the mean percentage of tumor in the prostate gland (the percentage of tumor on each slide divided by the number of slides from the prostate gland). Extraprostatic involvement was defined as tumor infiltration of the adipose tissue, the neurovascular plexus, or the parenchyma of the seminal vesicles. The TNM 2010 system was used for tumor staging and patients were classified as pT2 when tumor was confined to the organ and pT3 when EPE or seminal vesicles were infiltrated by tumor. PM was considered when tumor glands were inked with India ink.

Twenty-two (3.7\%) of the 592 patients exhibited insignificant tumor in their RP, which was defined as an organ-confined adenocarcinoma, Gleason score (GS) $\leq 6$ with no tertiary high grade Gleason pattern, and a tumor volume $\leq 0.5$ $\mathrm{cm}^{3}$ (Figure-1). The biopsies from these patients were analyzed, and twenty cases (91\%) presented the following features: adenocarcinoma $(\mathrm{GS}) \leq 6$, one to three positive cores that consisted of less than 50\% tumor and a total percentage of positive fragments $\leq 10 \%$. These features, which are similar to the Epstein criteria regarding pathological findings and are referred during the paper as "our criteria", served as the parameters for the selection of patients with potentially insignificant prostate cancer. In order to evaluate the importance of pathological findings in defining these tumors, our criteria were purposely created based solely on the biopsy features and did not include PSA or any imaging method. These criteria were used to review the biopsy and radical prostatectomy results and define the sensitivity, specificity, and positive and negative predictive value of these parameters for the identification of insignificant prostate carcinoma in our population.

\section{RESULTS}

Twenty-two (3.7\%) of the 592 patients exhibited clinically insignificant prostate cancer in their RP with a mean GS of 5.9 (range 5 to 6) and a tumor volume less than $0.5 \mathrm{~cm} 3$ that was organ-confined. Twenty patients (91\%) also had potentially insignificant prostate cancer in their biopsies. These biopsy features, which served as 
Figure 1 - Selection of patients who had clinically insignificant prostate cancer in radical prostatectomy as well as features of insignificant tumor in biopsy.

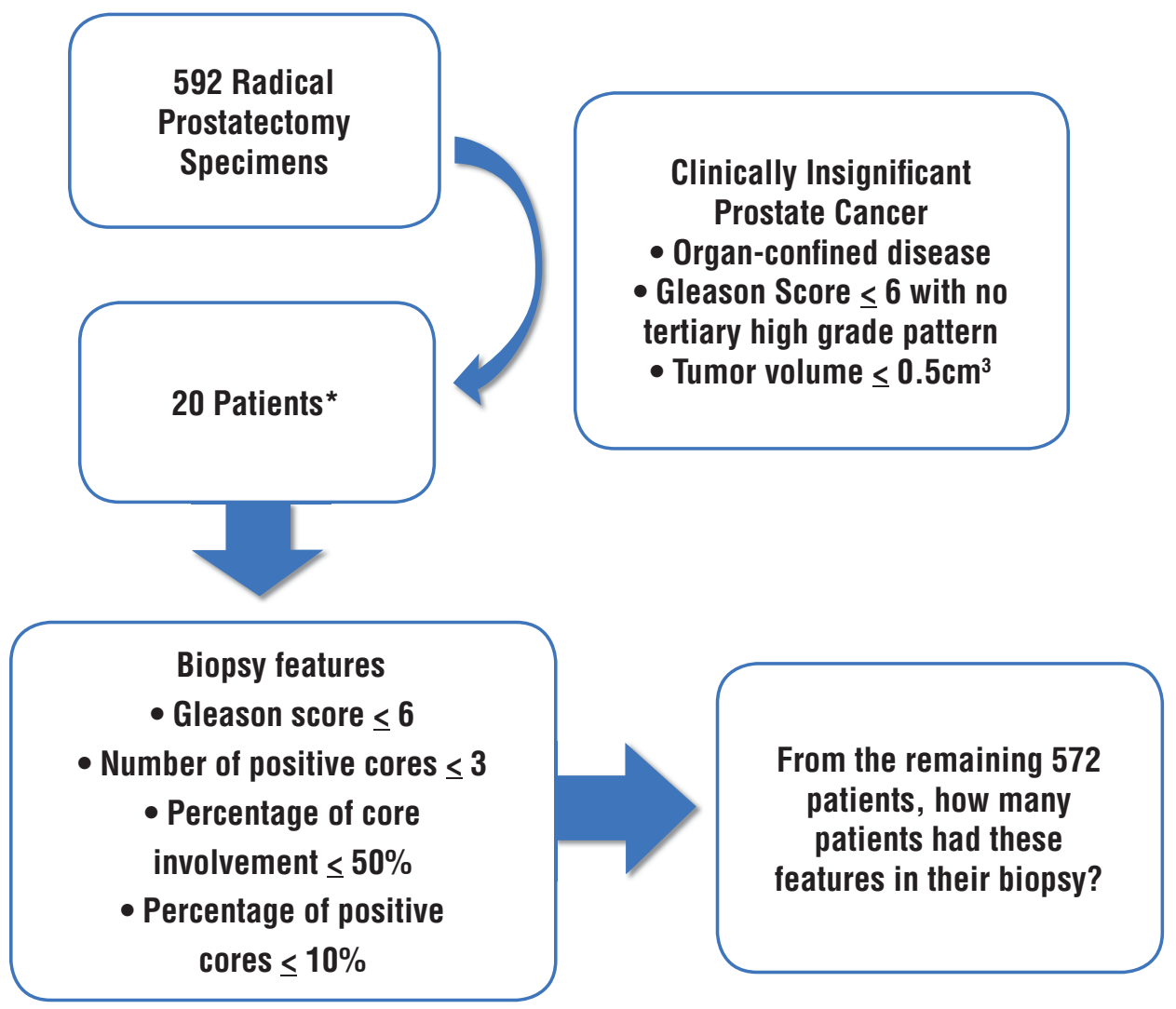

* 22 patients had clinically insignificant prostate cancer; however, 2 patients had unfavorable features in their biopsy.

our criteria mentioned above, exhibited a mean GS of 5.9 and mean positive cores of $6 \%$. This group was considered real positive (Table-1). The remaining 2 patients (9\%) had GS of 7 in the biopsy, 3 cores positive for tumor, a higher percentage of a single core (40\% and 70\%) and 4\% and 6\% positive cores (in 13 and 14 cores, respectively). We consider this group as false negative, since we would not expect them to have insignificant tumor in the RP. The RPs of additional patients who exhibited similar prostate biopsy characteristics of the real positive group were re-examined. A total of 149 patients (26\%) had biopsies that met our criteria and were characterized as probable IPC.
The mean GS was also 5.9, the mean percentage of tumor in a single core was $23.3 \%$, and the mean percentage of cores that were positive for tumor was $5.4 \%$ (range 1.5 to $10 \%$ ), which represented one to two positive cores in a mean of 15 biopsies. However, their RPs revealed clinically significant carcinomas, including patients with pT3 disease (4.7\%) as well as patients with intermediate and high grade tumor (56 patients with GS 7 and 16 with GS > 7). This group was considered false positive, which means they could have been considered as having IPC based on their biopsy features, despite having tumor with adverse pathological characteristics. The real negative group 
Table 1 - Comparison between biopsy and RP of the patients regarding clinical significance of the tumor.

\begin{tabular}{llll}
\hline Number of patients $(\%)$ & Biopsy & Radical Prostatectomy 1 & Real Positive \\
\hline $20(3.4 \%)$ & insignificant & insignificant & False Positive \\
$149(25.2 \%)$ & insignificant & significant & False Negative \\
$2(0.34 \%)$ & significant & insignificant & Real Negative \\
\hline
\end{tabular}

1 Gold Standard

was composed of 421 men whose biopsy and RP revealed significant prostate cancer. All patient data are detailed in Table- 2 .

The sensitivity of our criteria for the identification of clinically insignificant prostate cancer in RP was 91\% with 74\% specificity. The positive predictive value was only $12 \%$, and the negative predictive value was $99.5 \%$. Therefore, if our criteria were used to predict significant cancer, the probability of a patient exhibiting significant cancer would be almost certain at level of accuracy of 91.4\%.

\section{DISCUSSION}

Several studies have questioned the efficacy of diagnosing limited cancer by needle biopsy, and the possibility of predicting tumor extent at RP based on biopsies. The applicability and validity of the criteria and nomograms that are commonly used to predict insignificant prostate cancer have also been discussed. The current study designed a set of novel criteria that were based on our own data and restricted to morphological aspects without the consideration of clinical stage or tumor markers. Although clinical findings, such as PSA level and PSA density, were not used, the features of the biopsies from patients with insignificant tumors at RP were similar to the biopsy criteria in the literature, such as a GS $\leq 6$ and limited tumor extent on biopsy. The current study does not propose criteria or models for the prediction of insignificant tumor. Conversely, this study clarified the use of precise morphological findings of prostate biopsy in the identification of insignificant prostate cancer.
Our data for the prediction of organ-confined tumors are consistent with the literature. Bastian et al. updated the Epstein criteria, which are the most widely used criteria for the prediction of clinically insignificant prostate cancer, and demonstrated concordance with pathologically organ-confined disease and a favorable grade (GS 6) in $83.9 \%$ of patients (4). Although $91.6 \%$ of these patients had organ-confined disease, $7.6 \%$ had a GS of 7 or higher.

The validity of the Epstein criteria has been questioned in European men. In a study that evaluated 366 patients who fulfilled the contemporary Epstein criteria demonstrated a similar rate of organ-confined disease (91.7\%). But the percent of patients with a GS of 7 was substantially higher; $24 \%$ of patients had a GS of 7 at RP, which yielded lower overall accuracy (76\% vs. 84\%) (1). Unfortunately, these authors did not mention the number of patients with clinically insignificant tumor at RP. According to our criteria, 95.3\% of the tumors were organ-confined, but $48.3 \%$ were GS $\geq 7$ (37.6\% patients were GS 7 and 10.7\% were GS 8 or 9), which is substantially higher than the reported rate in previous studies.

Jeldres et al. argued that the differences between biopsy and radical prostatectomy with respect to GS contributed to the observed error rate of the Epstein criteria (1). A potential upgrading of the GS occurs in $24.3 \%$ to $28.2 \%$ of patients $(8,9)$. The grade assignment is also harder to predict because of the small amount of tumor that is analyzed on biopsy. High-grade prostate cancer is the most important predictor of prognosis (10) 


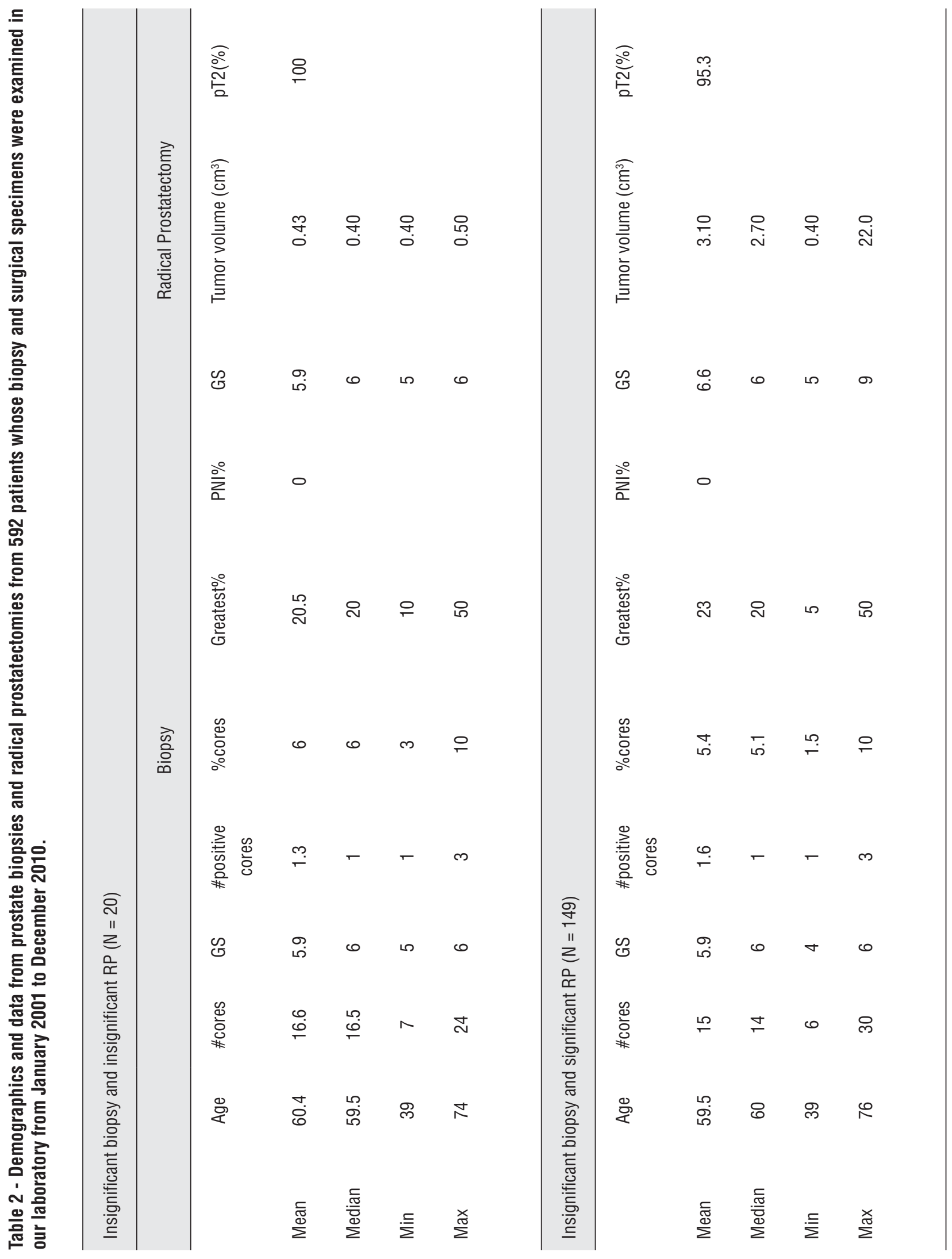




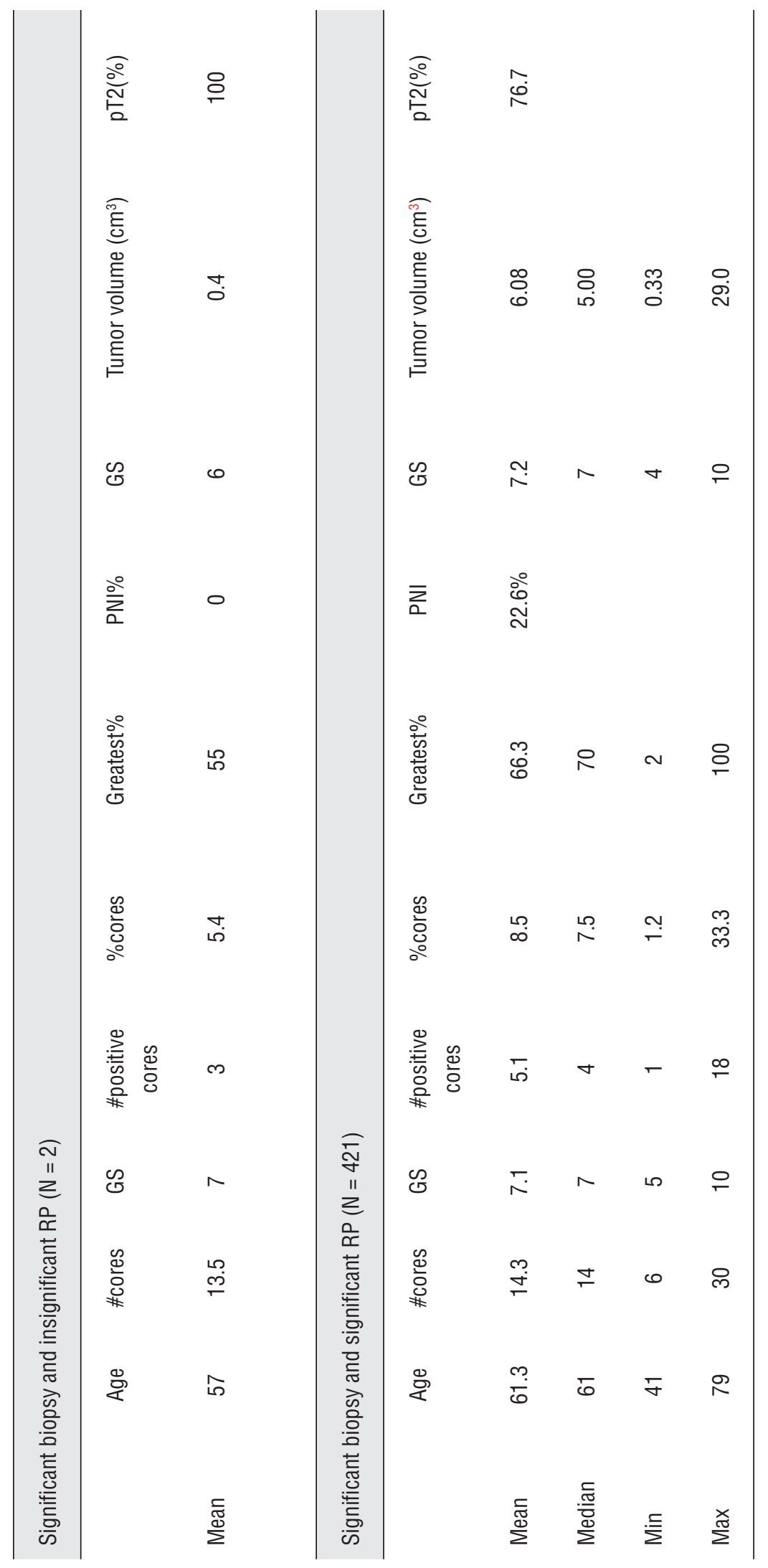


even when the tumor is organ confined, and it is the most informative predictor of biochemical recurrence (11).

Epstein et al. created their criteria to predict insignificant tumor in patients with stage T1c, and it accurately predicted $73 \%$ of insignificant tumor (3). The current study predicted only $12 \%$ of the clinically insignificant tumors, but our samples were not limited to stage T1c patients. Despite this low positive predictive value, the negative predictive value was 99\%, which indicated that ninety-nine percent of the tumors that were identified as significant and required active therapy were indeed significant tumors. This result addresses the concern of overdiagnosis, which occurs at a rate of approximately 56\% $(12,13)$.

The retrospective assessment of biopsies from patients with insignificant or significant tumors at RP revealed that all of the parameters that are usually used to estimate tumor extent, such as the number of positive cores, maximal involvement of a single core and the percentage of positive cores, were similar in both groups. These results suggest an absence of rules for insignificant tumor behavior on needle biopsy.

Numerous difficulties exist in the use of prostatic needle biopsies to predict limited cancer at RP, and even the smallest cancer focus on a needle biopsy does not guarantee a clinically insignificant tumor. Small amounts of carcinoma (total linear extent less than $3 \mathrm{~mm}$ ) do not predict insignificant tumors $(14,15)$. Patients who are diagnosed based on a single focus less than $3 \mathrm{~mm}$ (GS $\leq 6$ ) have only a 30\% chance of harboring an insignificant tumor (16). Samaratunga et al. examined 58 patients with a single minute focus of a GS 6 on biopsy, and only 10 patients (17\%) had insignificant tumor at RP (17). Forty-eight patients had significant tumor, 8 patients had extraprostatic extension and $32 \%$ of the patients had a GS > 6 . These authors concluded that a minute focus of prostate cancer on a needle biopsy is not indicative of insignificant carcinoma in most cases. Interestingly, this study was performed without PSA screening. However, these authors demonstrated that a larger prostate size was significantly correlated with potentially insignificant cancer. These patients likely presented earlier for PSA tes- ting because of symptoms, and an elevated PSA level due to the benign enlargement might lead to biopsies at an earlier stage. Cupp et al. demonstrated only an $8 \%$ risk of insignificant cancer using tumor volume at biopsy (14), which is similar to our results. The percentage of tumor extension in millimeters relative to the total extension of all of the cores was 5\% and a GS less than 7.

In contrast, Allan et al. evaluated 54 PSA-screened patients with limited adenocarcinoma ( $<0.5 \mathrm{~mm}$ ) on biopsy; the majority of these patients exhibited potentially insignificant cancer, but only one-third warranted definitive therapy (2). Potentially clinically insignificant tumors were present in $67 \%$ of the patients in this study, and $44 \%$ of these patients had small tumors at RP (less than $0.1 \mathrm{cc}$ ). These authors also reported that a PSA density cutoff of 0.15 or less was correlated with clinically insignificant tumor, and the association of these criteria with limited cancer on biopsy predicted patients with insignificant tumors with a greater than $80 \%$ accuracy.

The Epstein criteria are not perfectly accurate, but no alternatives for prediction of clinically insignificant prostate cancer are available (1). Kattan et al. derived several nomograms for the prediction of pathologically confirmed insignificant prostate cancer with an accuracy of 64 to $79 \%$ (18). However, this series included 13 to $20 \%$ of Gleason patterns of 2 as part of the GS, which is much lower than the GS consensus from 2005. Nakanishi et al. improved the accuracy of the existing tools to 73\%, especially in patients with a single positive core at biopsy (19).

Chun et al. developed a nomogram to predict the probability of insignificant prostate cancer in a cohort of 1132 men and revealed a predictive accuracy of 90\% (5). However, a strikingly important proportion of patients who were qualified with a high probability of insignificant tumor using the Chun et al. nomogram (63\%) and the Kattan et al. nomogram (45\%) harbored aggressive prostate cancer at RP. Chun et al. concluded that the nomogram studies were similar to the original Epstein et al. criteria in their ability to predict pathologically confirmed insignificant prostate cancer. Clinicians may expect a 80\% accuracy when organ-confined prostate cancer is predicted 
or a 76 to $79 \%$ accuracy when pathologically confirmed insignificant prostate cancer is predicted.

Do these data suggest that a significant number of patients might be left undertreated? Is active surveillance a dangerous choice that could jeopardize the curability of prostate cancer in some men? The answer to these questions appears to be no.

A review of active surveillance revealed that the majority of patients stay on active surveillance, and once a patient requires active treatment, that patient presents with curable prostate cancer (12). The detection of prostate cancer progression in a patient who is selected for active surveillance remains a continuing challenge, and the PSA level remains important during the decision process. The progression of Gleason grade and the increased percentage of cancers per core are also indicators for the cessation of active surveillance.

Duffield et al. have also studied the RP findings of patients in whom active surveillance has failed (13). These authors relied solely on subsequent biopsy pathology to determine progression, and more extensive disease was observed in surveillance biopsies during the first 2 years of follow-up in the majority of cases.

Despite their high accuracy rates, currently available models for the prediction of insignificant prostate cancer are incorrect in 10 to $20 \%$ of cases. The addition of novel markers is required, and current imaging techniques, such as multiparametric magnetic resonance, may have a potential role.

Tumor location is problematic for sampling adequately. Anterior tumors are difficult to assess and sample clinically, and the amount of these tumors in biopsies is lower than the amount of tumor from equivalently sized posterior tumors $(20,21)$. These tumors appeared smaller on biopsy, but they were also undersampled. Takashima et al. analyzed the anatomical patterns of disease distribution in nonpalpable tumors and demonstrated that these tumors were localized predominantly in the anterior half of the prostate at the apical to midprostate level (21). These authors suggested that additional cores from the anterior apical site could enhance the detection rate of prostate cancer. Miyake et al. evaluated the significance of additional cores in the dorsal apex and demonstrated a significant increase $(9.3 \%)$ in the cancer detection rate, particularly for early stage disease (22).

In conclusion, we have shown that the biopsy data exclusively are accurate to diagnose IPC, and pathologist should suggest this possibility in their reports helping urologists, oncologists and radiotherapists to choose the better treatment for each patient.

\section{CONFLICT OF INTEREST}

None declared.

\section{REFERENCES}

1. Jeldres C, Suardi N, Walz J, Hutterer GC, Ahyai S, Lattouf $\mathrm{JB}$, et al.: Validation of the contemporary epstein criteria for insignificant prostate cancer in European men. Eur Urol. 2008; 54: 1306-13.

2. Allan RW, Sanderson H, Epstein JI: Correlation of minute (0.5 MM or less) focus of prostate adenocarcinoma on needle biopsy with radical prostatectomy specimen: role of prostate specific antigen density. J Urol. 2003; 170: 370-2.

3. Epstein JI, Walsh PC, Carmichael M, Brendler CB: Pathologic and clinical findings to predict tumor extent of nonpalpable (stage T1c) prostate cancer. JAMA. 1994; 271 : 368-74.

4. Bastian PJ, Mangold LA, Epstein JI, Partin AW: Characteristics of insignificant clinical T1c prostate tumors. A contemporary analysis. Cancer. 2004; 101: 2001-5.

5. Chun FK, Haese A, Ahyai SA, Walz J, Suardi N, Capitanio $U$, et al.: Critical assessment of tools to predict clinically insignificant prostate cancer at radical prostatectomy in contemporary men. Cancer. 2008; 113: 701-9.

6. Epstein JI, Allsbrook WC Jr, Amin MB, Egevad LL; ISUP Grading Committee. The 2005 International Society of Urological Pathology (ISUP) Consensus Conference on Gleason Grading of Prostatic Carcinoma. Am J Surg Pathol. 2005; 29: 1228-42.

7. Humphrey PA, Vollmer RT: Intraglandular tumor extent and prognosis in prostatic carcinoma: application of a grid method to prostatectomy specimens. Hum Pathol. 1990; 21: 799-804.

8. Chun FK, Briganti A, Shariat SF, Graefen M, Montorsi F, Erbersdobler $A$, et al:: Significant upgrading affects a third of men diagnosed with prostate cancer: predictive nomogram and internal validation. BJU Int. 2006; 98: 329-34. 
9. Moreira Leite KR, Camara-Lopes LH, Dall'Oglio MF, Cury J, Antunes AA, Sañudo A, Srougi M: Upgrading the Gleason score in extended prostate biopsy: implications for treatment choice. Int J Radiat Oncol Biol Phys. 2009; 73: 353-6.

10. Stamey TA, McNeal JE, Yemoto CM, Sigal BM, Johnstone IM: Biological determinants of cancer progression in men with prostate cancer. JAMA. 1999; 281: 1395-400.

11. Chun FK, Briganti A, Jeldres C, Gallina A, Erbersdobler A, Schlomm T, et al.: Tumour volume and high grade tumour volume are the best predictors of pathologic stage and biochemical recurrence after radical prostatectomy. Eur $\mathrm{J}$ Cancer. 2007; 43: 536-43.

12. Bastian PJ, Carter BH, Bjartell A, Seitz M, Stanislaus P, Montorsi $F$, et al.: Insignificant prostate cancer and active surveillance: from definition to clinical implications. Eur Urol. 2009; 55: 1321-30.

13. Duffield AS, Lee TK, Miyamoto H, Carter HB, Epstein JI: Radical prostatectomy findings in patients in whom active surveillance of prostate cancer fails. J Urol. 2009; 182: 2274-8.

14. Cupp MR, Bostwick DG, Myers RP, Oesterling JE: The volume of prostate cancer in the biopsy specimen cannot reliably predict the quantity of cancer in the radical prostatectomy specimen on an individual basis. J Urol. 1995; 153: 1543-8.

15. Bruce RG, Rankin WR, Cibull ML, Rayens MK, Banks ER, Wood DP Jr: Single focus of adenocarcinoma in the prostate biopsy specimen is not predictive of the pathologic stage of disease. Urology. 1996; 48: 75-9.
16. Boccon-Gibod LM, Dumonceau 0, Toublanc M, Ravery V, Boccon-Gibod LA: Micro-focal prostate cancer: a comparison of biopsy and radical prostatectomy specimen features. Eur Urol. 2005; 48: 895-9.

17. Samaratunga H, Yaxley J, Kerr K, McClymont K, Duffy D: Significance of minute focus of adenocarcinoma on prostate needle biopsy. Urology. 2007; 70: 299-302.

18. Kattan MW, Eastham JA, Wheeler TM, Maru N, Scardino PT, Erbersdobler A, et al.: Counseling men with prostate cancer: a nomogram for predicting the presence of small, moderately differentiated, confined tumors. J Urol. 2003; 170: $1792-7$.

19. Nakanishi H, Wang $X$, Ochiai A, Trpkov K, Yilmaz A, Donnelly JB, et al.: A nomogram for predicting low-volume/ low-grade prostate cancer: a tool in selecting patients for active surveillance. Cancer. 2007; 110: 2441-7.

20. Bott SR, Young MP, Kellett MJ, Parkinson MC; Contributors to the UCL Hospitals' Trust Radical Prostatectomy Database: Anterior prostate cancer: is it more difficult to diagnose? BJU Int. 2002; 89: 886-9.

21. Takashima R, Egawa S, Kuwao S, Baba S: Anterior distribution of Stage T1c nonpalpable tumors in radical prostatectomy specimens. Urology. 2002; 59: 692-7.

22. Miyake H, Harada K, Inoue TA, Takenaka A, Hara I, Fujisawa M: Additional sampling of dorsal apex on systematic prostate biopsy: impact on early detection of prostate cancer. Urology. 2007; 69: 738-42.

\footnotetext{
Correspondence address:

Dr. Katia Ramos Moreira Leite Rua Adma Jafet 91

Sao Paulo, SP, 01308-050, Brazil Fax: +55 $113155-3836$

E-mail: katiaramos@uol.com.br
} 